\title{
Island tameness: An altered cardiovascular stress response in Galápagos marine iguanas
}

\author{
Maren N. Vitousek ${ }^{\text {a,b, },}$, L. Michael Romero ${ }^{c}$, Elisa Tarlow ${ }^{\text {b }}$, Nicole E. Cyr ${ }^{c}$, Martin Wikelski ${ }^{\text {a,b }}$ \\ a Department of Ecology and Evolutionary Biology, Princeton University, Guyot Hall, Princeton, NJ 08544-2016, USA \\ ' Max Planck Institute for Ornithology, Volgelwarte Radolfzell, Schlossallee 2, D-78315 Radolfzell, Germany \\ ' Department of Biology, Tufts University, 163 Packard Avenue, Medford, MA 02155, USA
}

\section{Keywords:}

Island tameness

Anti-predator behavior

Stress response

Heart rate

Catecholamines

Corticosterone

Epinephrine

Galápagos marine iguana

Amblyrhynchus cristatus

\begin{abstract}
A B S T R A C T
Island tameness is a widely documented phenomenon in which island species, particularly those that have evolved with no or few natural predators, show a greatly reduced behavioral response when faced with unfamiliar predators. This insufficient anti-predator response has led to widespread population declines among many island species exposed to novel predators, and has become a serious conservation problem. Despite its prevalence, the underlying physiology of island tameness is not known. Here we report that although Galápagos marine iguanas (Amblyrhynchus cristatus) initiated flight from an evolutionarily recent and unfamiliar potential predator (humans), they failed to show the cardiovascular stress response that facilitates successful escape, even after a prior capture experience. In contrast, when approached by a native predator (the Galápagos hawk; Buteo galapagoensis), marine iguanas show markedly increased heart rate independent of initiating escape movement. The secretion of catecholamines appears to be central to the initiation of escape behavior: naïve animals remotely injected with epinephrine immediately increased flight initiation distance, whereas those injected with corticosterone did not. Our results provide the first evidence that muted escape behavior in predator-naïve species is indicative of both a cognitive deficit in recognizing potential predators and a catecholamine deficit in response. Understanding how the response to predators differs in predator-naïve species could enable the design of maximally effective techniques for inducing an anti-predator response in these vulnerable species.
\end{abstract}

\section{Introduction}

Many native species face difficulties in adapting to novel predators [1-3]. Particularly at risk are those that have spent much of their evolutionary history isolated from all or most major suites of predators $[4,5]$. These species often lack generalized anti-predator behavior, a phenomenon known as 'island tameness'. Although decreased wariness can be adaptive in an environment in which novel threats are exceedingly rare, the inability to recognize and respond appropriately to introduced predators has resulted in severe population declines in a variety of species [6-8].

Attempts to train island species to respond appropriately to introduced predators have met with limited and variable success. Several studies have shown that classical conditioning (pairing a model of an unfamiliar predator with an unpleasant stimulus) can induce an escape response during subsequent presentations of the model [9-12]. At least in some species, however, this acquired escape

\footnotetext{
* Corresponding author. Present address: Department of Ecology and Evolutionary Biology, University of Colorado Boulder, UCB 334, Boulder, CO 80309-0334, USA Tel.: + 1609468 5443; fax: + 1303 492-8699.

E-mail address: maren.vitousek@colorado.edu (M.N. Vitousek).
}

behavior is insufficient to avoid predation. The flight distance of Galápagos marine iguanas (Amblyrhynchus cristatus) increases after exposure to unfamiliar predators, but remains an order of magnitude too low to enable successful escape $[13,14]$.

Although the absence of a generalized anti-predator response in evolutionarily naïve species is a well-documented phenomenon, it is not known whether or how the physiological stress response differs in these species. The normal physiological stress response is highly conserved across taxa and consists of coordinated cardiovascular and endocrine elements that are considered vital for surviving challenges [15-17]. A fundamental component of this response is the rapid secretion of catecholamines that trigger an elevation in heart rate and stroke volume in preparation for extreme physical exertion.

We studied the relationship between anti-predator behavior and the initiation of a cardiovascular stress response in the classically tame Galápagos marine iguana. Adult iguanas face no native terrestrial predators, and only a single aerial predator: the Galápagos hawk (Buteo galapagoensis). Relatively recent introductions of novel terrestrial predators (feral dogs and cats) have resulted in the widespread decline of marine iguana populations on inhabited islands. Although marine iguanas attempt to evade novel predators, this behavioral response is largely unsuccessful: affected populations 
can suffer $27 \%$ annual mortality from feral dogs [18], and recruitment on some islands with introduced predators approaches zero [19].

\section{Methods}

\subsection{Experiment 1}

We studied the cardiovascular stress response of 11 adult male marine iguanas on the island of Santa Fe, Galápagos $\left(90^{\circ} 2^{\prime} \mathrm{W}, 0^{\circ} 50^{\prime} \mathrm{S}\right)$, in July of 2003. Santa Fe is not inhabited by humans or introduced predators, and visual encounters with humans prior to the start of the study are likely to have been rare: iguanas are highly site faithful [20] and the present study site is inaccessible to tourists and separate from our long-term study colony. Study males were implanted with miniaturized data loggers that recorded heart rate at $2 \mathrm{~s}$ intervals. Iguanas were hand-captured and anesthetized with a mixture of isoflurane and air. Isoflurane does not appear to cause retrograde amnesia in vertebrates $[21,22]$; thus, it seems unlikely that this process affected the iguanas' memory of their initial capture. The surgical site was prepared using aseptic techniques, and the data logger ( $\sim 15 \mathrm{~g} ; \leq 1 \%$ of male body weight) inserted into the coelomic cavity through a $4-5 \mathrm{~cm}$ paramedian incision initiated just caudal to the xiphoid process. Loggers were anchored to the body wall with two silk sutures (\#2, Dexon, Germany), and the incision was closed using a horizontal mattress pattern (body wall: Chromic Gut \#1, Dexon, Germany, skin: Silk Suture \#2). Iguanas were held in a warm location during recovery, released at the location of capture and allowed to recuperate for several days. At the conclusion of the study, data loggers were removed using the same surgical techniques.

Marine iguanas do not appear to suffer major post-surgical trauma. Previous studies found that implanted and control marine iguanas do not differ in corticosterone levels $24 \mathrm{~h}$ after surgery [23], and show indistinguishable active mate choice behavior five days post-surgery [20]. No mortality occurred during our study, and data logger implantation has no effect on annual survival rates [20]. All protocols used in this study adhered to the Animal Behavior Society Guidelines for the Use of Animals in Research, and were approved by the IACUC Committees of Princeton University and Tufts University.

In order to measure the cardiovascular stress response of marine iguanas to novel threats we conducted a series of simulated predator approaches using an evolutionarily recent and unfamiliar potential predator (hereafter referred to as an unfamiliar predator): humans. During these trials focal individuals were directly approached from a distance of $10-12 \mathrm{~m}$ at a rate of $\sim 1.3 \mathrm{~m} / \mathrm{min}$ until the iguana initiated escape movement or the experimenter was $1.8 \pm 0.1 \mathrm{~m}$ away. In order to standardize the appearance and approach speed of the unfamiliar predator, all approaches were conducted by the same experimenter $[13,24]$. This individual (M.W.) had also hand-captured all iguanas prior to data logger implantation; thus all animals had previously had a stressful experience with the experimenter. Each iguana's mean heart rate during approaches was compared with baseline values recorded 3 to $5 \mathrm{~min}$ prior to the initiation of the trial. Two individuals were excluded due to incomplete data logger recordings.

Heart rate and anti-predator behavior during naturally occurring over-flights of Galápagos hawks were recorded during eight days of continual observation. Hawks maintained a much greater distance from the iguanas $(\sim 20 \mathrm{~m})$ than approaching humans $(1.8 \mathrm{~m})$. Flying hawks also traveled more quickly. However, approach speed does not appear to affect behavioral response: a similar proportion of marine iguanas initiated flight when approached by real hunting hawks (traveling at $\sim 500-700 \mathrm{~m} / \mathrm{min}$ ) and hawk models flown overhead at a speed of $\sim 6 \mathrm{~m} / \mathrm{min}(t=1.46, d f=10, p=0.175)$. Likewise, independent of approach speed, iguanas appear able to distinguish between the threat posed by different species: a significantly higher proportion of marine iguanas exhibited escape behavior when approached by a hawk model than by a frigate bird model traveling at the same speed $(t=31.11, d f=10, p<0.001)$. In the current experiment, none of the individuals with data loggers were directly targeted by hawks during the study period, although hawks were frequently hunting in the area. Although study animals alerted to the presence of approaching hawks, because they were not directly targeted, they did not initiate escape movement. In order to enable a comparison of heart rate during escape from known vs. unfamiliar predators we have included heart rate data from seven separate individuals escaping from hawk attacks (generated during a longterm study of marine iguanas on Santa Fe Island).

\subsection{Experiment 2}

We tested how epinephrine and corticosterone influence the initiation of escape behavior by manipulating hormone levels in naïve (not previously captured) iguanas using remote injections. Large males of approximately the same size and who were resting in the sun were randomly targeted in January of 2007. Each animal was injected using one of two methods: a pole syringe or a syringe and needle delivered using a blowgun that injected its dose upon impact (both devices from Animal Care Equipment and Services, Boulder $\mathrm{CO}$ ). Under both methods we approached the target animal to within $1-3 \mathrm{~m}$ and delivered the dose intramuscularly to the base of the tail. After injection animals typically moved off a few meters and resumed sunning behavior. We then monitored behavior for the next $10 \mathrm{~min}$, but no animal took more than 4 steps, and most remained stationary throughout this period. At the end of the $10 \mathrm{~min}$ observation period we approached the animal from a distance of $10-12 \mathrm{~m}$ at approximately $20 \mathrm{~m} / \mathrm{min}$ and recorded the distance at which the animal initiated escape behavior (the flight initiation distance). After determining flight initiation distance, each iguana was captured by hand.

Iguanas were injected with $200 \mu \mathrm{l}$ of either $0.33 \mathrm{mg} / \mathrm{kg}$ of epinephrine (Sigma) dissolved in distilled water or $1 \mathrm{mg} / \mathrm{kg}$ of corticosterone (Sigma) dissolved in peanut oil. Controls were injected with $200 \mu$ l of distilled water. Each treatment group contained 8 animals, for a total of 24. Because individual iguanas could not be weighed prior to injection, doses were calculated on the basis of an estimated $1.5 \mathrm{~kg}$ animal. When weighed after capture, there was no systematic bias between groups and each mean was close to $1.5 \mathrm{~kg}(1.60 \pm 0.07,1.52 \pm 0.08$, and $1.53 \pm$ $0.08 \mathrm{~kg}$ for water, epinephrine, and corticosterone treatments, respectively; $F(2,21)=0.34, p=0.72$ ).

Immediately upon capture, each iguana was bled using a $2 \mathrm{ml}$ heparinized vacutainer syringe (Becton-Dickinson). Blood was collected from the caudal vein, centrifuged, and the plasma removed and stored cold [25]. Plasma was transported to Tufts University and assayed for corticosterone using a radioimmunoassay reported previously [25]. All samples were measured in a single assay, with an intraassay variability of $2.9 \%$.

\subsection{Statistical analysis}

Statistical analyses were conducted using SPSS 15.0.1 (Experiment 1) and JMP (SAS) 5.0.1.2 (Experiment 2). Data conformed to assumptions of normality and homogeneity of variances. Differences in heart rate prior to and during escape behavior were investigated with paired samples $t$-tests. Effect sizes (Cohen's $d$ ) were calculated to provide a measure of the strength of the relationship between variables. The effect of approach distance on heart rate (prior to the initiation of escape behavior) during unfamiliar predator simulations was investigated with repeated measures ANOVA. Mauchly's test indicated that the assumption of sphericity had been violated, $\chi^{2}(20)=$ 42.97, $p<0.05$, therefore degrees of freedom were corrected using Greenhouse-Geisser estimates of sphericity $(=0.44)$. The effect of epinephrine and corticosterone injections on plasma corticosterone concentrations, flight initiation distances, and weight were investigated with factorial ANOVA. 


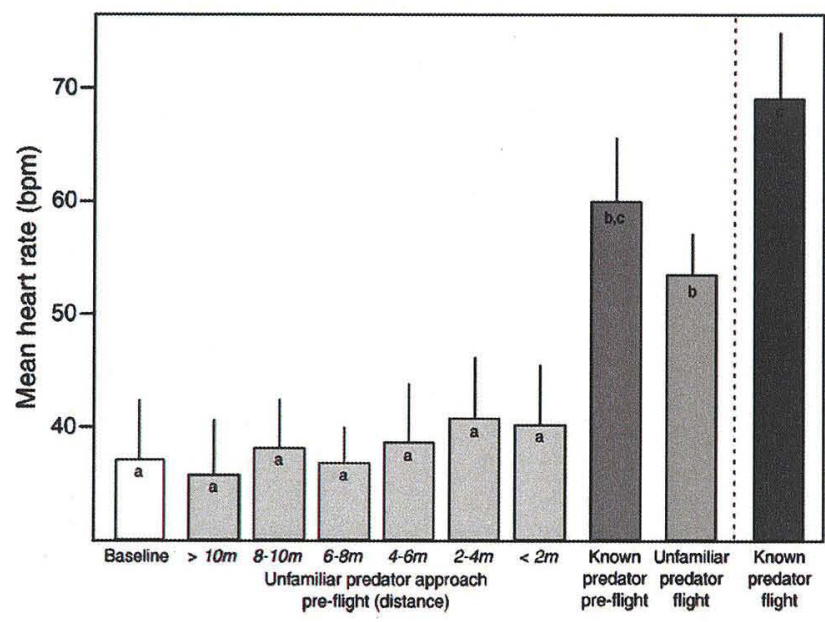

Fig. 1. Unfamiliar predators elicit an altered physiological stress response in marine iguanas. When approached by an evolutionarily recent and unfamiliar potential predator (humans), marine iguanas do not increase heart rate prior to initiating escape movement, regardless of proximity. Heart rate does increase, independent of flight initiation, when approached by a known predator (the Galápagos hawk). Heart rate is elevated during flight from both known and unfamiliar predators, although the magnitude is greater for known predators. Bars represent means (SE); $n=9$ for all categories except 'known predator flight', where $n=7$.

\section{Results}

\subsection{Experiment 1}

When approached by a Galápagos hawk, marine iguanas immediately elevated heart rate over baseline levels (Fig. 1; paired samples $t$-test, $t=-3.56, d f=8, p=0.007, d=1.39$ ). This response was independent of the initiation of escape behavior, and is indicative of epinephrine release upon visual contact with a known predator. During approaches by an unfamiliar predator with which they had a prior stressful experience (capture and handling) marine iguanas failed to display an elevation in heart rate above baseline prior to flight, regardless of proximity to the approaching observer (repeated measures ANOVA, $F(2.6,21.1)=0.71, p=0.543, \eta_{p}^{2}=0.081$ ).

Marine iguanas increased heart rate during flight from unfamiliar predators (paired samples $t$-test, $t=-3.13, d f=8, p=0.014$, $d=1.26$ ), although heart rate remained lower than during flight

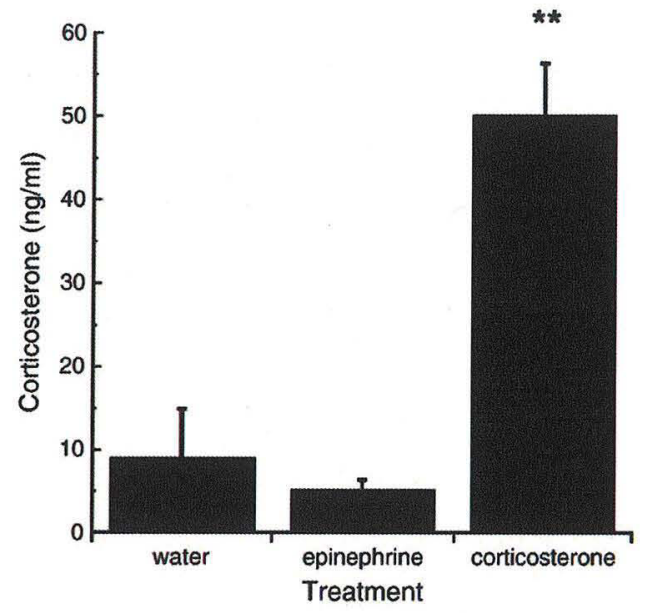

Fig. 2. Hormone levels following remote injection. Iguanas remotely injected with corticosterone showed elevated levels of circulating corticosterone $10 \mathrm{~m}$ after injection (ANOVA, $F(2,21)=5.21, p<0.01$ ). Epinephrine-injected iguanas had corticosterone levels similar to water-injected controls.

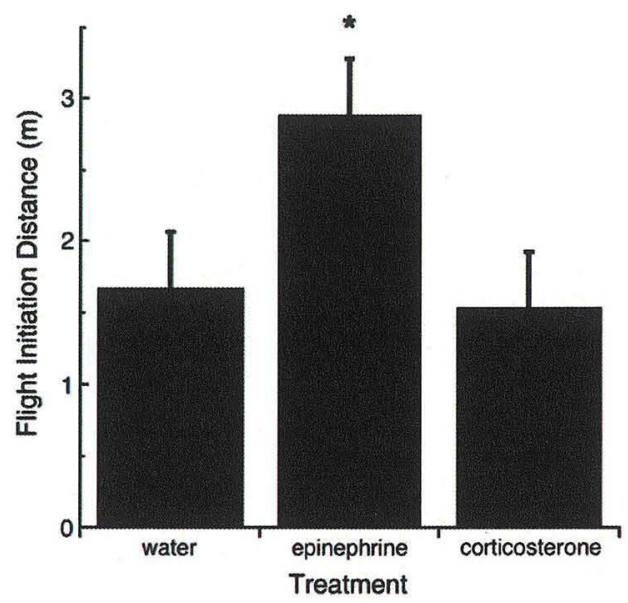

Fig. 3. Epinephrine increases flight initiation distance. Naïve marine iguanas increased flight initiation distance following remote injection with epinephrine (ANOVA, $F(2,21)=$ $3.64, p<0.044$ ), but not with corticosterone.

from known predators (independent samples $t$-test, $t=-2.40$, $d f=14, p=0.031, d=1.29$ ). Iguanas initiated escape movement during most approach trials, but this response appears to be insufficient to avoid predation, as all marine iguanas were easily hand-captured at the conclusion of the study.

\subsection{Experiment 2}

The remote injection procedure was successful: iguanas administered corticosterone had significantly higher levels of circulating corticosterone when captured $10 \mathrm{~min}$ after injection than those injected with water or epinephrine (ANOVA, $F(2,21)=5.21, p<0.01$; Fig. 2). Naïve marine iguanas remotely injected with epinephrine significantly increased their wariness, exhibiting greater flight initiation distances than those injected with water or corticosterone (ANOVA, $F(2,21)=$ $3.64, p<0.044$; Fig. 3).

\section{Discussion}

Our findings indicate that the physiological stress response of marine iguanas differs when faced with a known vs. an evolutionarily recent and unfamiliar potential predator (hereafter referred to as an unfamiliar predator). The typical vertebrate stress response includes the secretion of catecholamines (epinephrine and norepinephrine), that increase heart rate and prepare the musculoskeletal system for increased energetic demands, and the activation of the hypothalamicpituitary-adrenal (HPA) axis, which culminates in the secretion of glucocorticoids (e.g. corticosterone or cortisol $[15,16])$. This response is immediate, consistent, and highly conserved across taxa, and is vital to successful escape from a predator $[26,27]$.

Although marine iguanas display a rapid increase in heart rate when approached by a known predator, they fail to show this response when approached by an unfamiliar predator, despite a prior capture experience. Thus, although marine iguanas are physiologically capable of generating an appropriate cardiovascular stress response to the threat of predation, they appear to be unable to recognize unfamiliar species as potential predators (even following a capture experience), and thus fail to secrete catecholamines in response to their approach.

In order to test the behavioral effects of catecholamines and glucococorticoids we remotely injected marine iguanas with epinephrine or corticosterone and measured subsequent flight initiation distance. The injection of epinephrine (but not corticosterone) resulted in an almost two-fold increase in flight initiation distance in naïve animals. This indicates that epinephrine is central to the 
generation of an appropriate escape response, impacting wariness and escape behavior even in the absence of experience. Even after epinephrine injection, however, the behavioral response of marine iguanas was modest in comparison to other iguanids [24] and did not prevent subsequent capture by the human "predator". The ability to recognize predators may therefore also play an important role in generating an appropriate behavioral response. The hormone manipulation and heart rate data suggest two changes in these animals: a cognitive deficit in identifying potential predators and a physiological damping that prevents the initiation of a sufficient escape response. To our knowledge, this study provides the first evidence that predator-naïve species display a fundamentally altered cardiovascular stress response when faced with unfamiliar predators.

Although we found that a single capture experience did not induce a subsequent heart rate response to unfamiliar predators, it is possible that many repetitions of this exposure would ultimately induce an appropriate behavioral and physiological stress response. Marine iguanas do exhibit a statistically significant (although minimal) increase in flight initiation distance after a single capture experience [13]. Populations on islands with more introduced predators also show greater wariness; however, their flight initiation distance remains two- to five-fold lower than in their mainland relatives [14], and mortality from introduced predators is substantial [18,19]. Thus, it appears that the learning process proceeds extremely slowly at best, requiring multiple exposures to unfamiliar predators. In a natural situation repeated exposures are unlikely; organisms that do not mount a strong physiological and behavioral response to the presence of a predator that had captured them previously would be unlikely to survive a second encounter.

Previous research has shown that while naïve marine iguanas do not raise glucocorticoid levels in response to human approach, a single capture experience is sufficient to restore a glucocorticoid stress response during subsequent approaches [13]. This contrasts with our finding that previously captured individuals maintained a damped cardiovascular stress response to human approach. Taken together, these results suggest that marine iguanas do not simply down-regulate their entire physiological stress response, but have uncoupled and differentially suppressed individual components of the stress response [28]. The reason for this differential suppression is not currently known. It is possible that the cardiovascular response may be more tightly regulated due to the high energetic cost of an increase in heart rate; alternatively, glucocorticoid release in response to stressors may be generally less plastic in vertebrates. This latter possibility has gained some support from work in European starlings (Sturnus vulgaris) showing that a variety of stressors elicit a similar glucocorticoid stress response but slightly different magnitudes of cardiovascular stress response $[28,29]$. Based on the high proportion of island endemics exhibiting an insufficient behavioral response to novel predators, we predict that the physiological stress response of other predator-naïve species may show alterations similar to those observed in marine iguanas. Future research on the stress physiology of other vulnerable species is necessary in order to test this prediction.

The hormone manipulation experiments that we conducted tested the immediate, nongenomic behavioral effects of epinephrine and corticosterone. Corticosterone rises to near-maximal levels within 15$30 \mathrm{~min}$ of the onset of an acute stressor, or almost immediately following corticosterone injection; the direct behavioral effects of this increase should be detectable by $10 \mathrm{~min}$ after injection. Corticosterone may also have genomic effects that would not have been detectable in our study, as they occur over a longer time scale (hours to days). However, these effects appear to have at most a weak influence on antipredator behavior in marine iguanas. Capture and restraint stress (which induces near-maximal corticosterone secretion) results in only a minimal increase in subsequent flight initiation distance in Galápagos marine iguanas [13], suggesting that any genomic effects of acute corticosterone secretion do not have a strong impact on wariness.
Because the design of this study compared naturally occurring hawk predation attempts with simulated approaches by an unfamiliar terrestrial predator, there were differences in the manner of predator approach. Although hunting hawks traveled more quickly than the experimenter in the unfamiliar predator simulations, approach speed does not appear to be a major factor in the response of marine iguanas: a similar proportion of individuals displayed an escape response to hunting hawks (traveling at $\sim 500-700 \mathrm{~m} / \mathrm{min}$ ), and hawk models flown overhead at $\sim 6 \mathrm{~m} / \mathrm{min}$. Marine iguanas were also approached more closely during the unfamiliar predator trials $(1.8 \mathrm{~m})$ than they were by hawks $(\sim 20 \mathrm{~m})$ during the study period. Despite this significant difference, marine iguanas showed a much stronger heart rate response to hawks.

Marine iguanas have no native terrestrial predators capable of taking adults, and face no introduced aerial predators. It is therefore not possible to compare the response of iguanas to known vs. unfamiliar predators that use the same method of approach. Some of the difference in the responsiveness of marine iguanas to hawks vs. humans may thus be due to the method of approach (aerial vs. terrestrial [30,31, but see 32]). However, the complete lack of a preparatory heart rate response when marine iguanas are faced with an unfamiliar terrestrial predator (with which they had a prior negative experience) indicates that their cardiovascular stress response is greatly modified from the typical - and highly conserved vertebrate stress response. This failure to mount a cardiovascular stress response may explain the insufficient behavioral response of marine iguanas to introduced terrestrial predators, and the high rates of mortality that result.

Understanding the physiological basis of island tameness may enable conservation managers to predict which species are particularly vulnerable to novel predators, and to develop successful techniques for inducing an anti-predator response in naïve species (e.g. pairing exogenous epinephrine with novel predator presentations). In general, we predict that evolutionarily naïve species do not release epinephrine when approached by an unfamiliar potential predator (an extremely rare event), but instead restrict this energetically costly cardiovascular response to the detection of known predators. This altered physiological response, combined with a cognitive deficit in recognizing unfamiliar species as predators, could explain why many island species have difficulty adapting to introduced predators.

\section{Acknowledgements}

We would like to thank J. Adelman, D. Blumstein, M. Hau, M. Niemack, R. Safran, K. Vitousek, D. Wilcove, and two anonymous reviewers for helpful comments on previous versions of the manuscript, A. Woakes for providing the data loggers, and G. Florant for help in the field. This work was supported by the National Science Foundation (DEB 0545744 and DEB 0545592), and Princeton University. The Charles Darwin Research Station and the Galápagos National Park Service provided permission and assistance. This is a contribution of the Charles Darwin Research Station.

\section{References}

[1] Case TJ, Bolger DT. The role of introduced species in shaping the distribution and abundance of island reptiles. Evol Ecol 1991;5:272-90.

[2] Fritts TH, Rodda GH. The role of introduced species in the degradation of island ecosystems: a case history of Guam. Annu Rev Ecol Syst 1998;29:113-40.

[3] Salo P, Korpimäki E, Banks PB, Nordström M, Dickman CR. Alien predators are more dangerous than native predators to prey populations. Proc R Soc Lond $B$ 2007;274:1237-43.

[4] Magurran AE. The causes and consequences of geographic variation in antipredator behavior: perspectives from fish populations. In: Foster SA, Endler JA, editors. Geographic variation in behavior: perspectives on evolutionary mechanisms. New York: Oxford University Press; 1999. p. 139-63.

[5] Blumstein DT, Daniel JC. The loss of anti-predator behaviour following isolation on islands. Proc R Soc Lond B 2005;272:1663-8. 
[6] Burbidge AA, Manly BF]. Mammal extinctions on Australian islands: causes and conservation implications. J Biogeogr 2002;29:465-73.

[7] Blackburn TM, Cassey P, Duncan RP, Evans KL, Gaston K]. Avian extinctions and mammalian introductions on Oceanic islands. Science 2004;305:1955-8.

[8] Clavero M, García-Berthou E. Invasive species are a leading cause of animal extinctions. Trends Ecol Evol 2006;20:110.

[9] McLean IG, Lundie-Jenkins G, Jarman PJ. Teaching an endangered mammal to recognize predators. Biol Conserv 1996;75:51-62.

[10] Griffin AS, Blumstein DT, Evans CS. Training captive-bred or translocated animals to avoid predators. Cons Biol 2000;14:1317-26.

[11] Griffin AS, Evans CS, Blumstein DT. Learning specificity in acquired predator recognition. Anim Behav 2001;62:577-89.

[12] Shier DM, Owings DH. Effects of predator training on behavior and post-release survival of captive prairie dogs (Cynomys ludovicianus). Biol Conserv 2006;132: 126-35.

[13] Rödl T, Berger S, Romero LM, Wikelski M. Tameness and stress physiology in a predator-naïve island species confronted with novel predation threat. Proc R Soc predator-naive island species

[14] Berger S, Wikelski M, Romero LM, Kalko EKV, Rödl T. Behavioral and physiological adjustments to new predators in an endemic island species, the Galápagos marine iguana. Horm Behav 2007; 52:653-63.

[15] Wingfield JC, Hunt L, Breuner C, Dunlap K, Fowler GS, Freed L, et al. Environmental stress, field endocrinology, and conservation biology. In: Clemmons JR, Buchholz $\mathrm{R}$, editors. Behavioral approaches to conservation in the wild. Cambridge: R, editors. Behavioral approaches to cons
Cambridge University Press; 1997. p. 95-131.

[16] Romero LM. Physiological stress in ecology: lessons from biomedical research. Trends Ecol Evol 2004;19:249-55.

[17] Blas J, Bortolotti GR, Tella JL, Baos R, Marchant TA. Stress response during development predicts fitness in a wild, long-lived vertebrate. Proc Natl Acad Sci USA 2007:104:8880-4.

[18] Kruuk H, Snell H. Prey selection by feral dogs from a population of marine iguanas (Amblyrhynchus cristatus). J Appl Ecol 1981;18:197-204.

[19] Cayot LJ, Rassman K, Trillmich F. Are marine iguanas endangered on islands with introduced predators? Not Galápagos 1994;53:13-5

[20] Vitousek MN, Mitchell MA, Woakes AJ, Niemack MD, Wikelski M. High costs of female choice in a lekking lizard. PLoS ONE 2007;2:e567.
[21] Dutton RC, Maurer AJ, Sonner JM, Fanselow MS, Laster MJ, Eger III E. Isoflurane causes anterograde but not retrograde amnesia for Pavlovian fear conditioning. Anesthesiology 2002;96(5):1223-9.

[22] Rau V, Oh I, Laster M, Eger III E, Fanselow MS. Isoflurane suppresses stressenhanced fear learning in a rodent model of post-traumatic stress disorder. Anesthesiology 2009;110(3):487-95.

[23] Butler PJ, Frappell PB, Wang T, Wikelski M. The relationship between heart rate and rate of oxygen consumption in Galápagos marine iguanas (Amblyrhynchus cristatus) at two different temperatures. J Exp Biol 2002;205:1917-24

[24] Burger J. Gochfeld M. Risk discrimination of direct versus tangential approach by basking black iguanas (Ctenosaura similis): variation as a function of human exposure. J Comp Psychol 1990;104:388-94.

[25] Romero LM, Wikelski M. Corticosterone levels predict survival probabilities of Galápagos marine iguanas during El Niño events. Proc Natl Acad Sci USA 2001;98: 7366-70.

[26] Wingfield JC. Modulation of the adrenocortical response to stress in birds. In: Davey KG, Peter RE, Tobe SS, editors. Perspectives in comparative endocrinology. Ottawa: National Research Council Canada; 1994. p. 520-8.

[27] Sapolsky RM, Romero LM, Munck AU. How do glucocorticoids influence stress responses? Integrating permissive, suppressive, stimulatory, and preparative actions. Endocr Rev 2000;21:55-89.

[28] Nephew BC, Kahn SA, Romero LM. Heart rate and behavior are regulated independently of corticosterone following diverse acute stressors. Gen Comp Endocrinol 2003;133:173-80

[29] Dickens MJ, Nephew BC, Romero LM. Captive European starlings (Sturnus vulgaris) in breeding condition show an increased cardiovascular stress response to intruders. Phys Biochem Zool 2006;79:937-43.

[30] Macedonia JM, Evans CS. Variation among mammalian alarm call systems and the problem of meaning in animal signals. Ethology 1993:93:177-97.

[31] Warkentin KJ, Keeley ATH, Hare JF. Repetitive calls of juvenile Richardson's ground squirrels (Spermophilus richardsonii) communicate response urgency. Can J Zool 2001;79:569-73.

[32] Templeton CN, Greene E, Davis K. Allometry of alarm calls: black-capped chickadees encode information about predator size. Science 2005;308:1934-7. 